\title{
Multimodal Fluorescence-Mediated Tomography and SPECT/CT for Small-Animal Imaging
}

\author{
Metasebya Solomon ${ }^{1,2}$, Ralph E. Nothdruft ${ }^{2}$, Walter Akers ${ }^{2}$, W. Barry Edwards ${ }^{2}$, Kexian Liang ${ }^{2}$, \\ Baogang Xu ${ }^{2}$, Gail P. Suddlow ${ }^{2}$, Hamid Deghani ${ }^{3}$, Yuan-Chuan Tai ${ }^{2}$, Adam T. Eggebrecht ${ }^{2}$, \\ Samuel Achilefu ${ }^{1,2,4}$, and Joseph P. Culver ${ }^{1,2,5}$ \\ ${ }^{I}$ Department of Biomedical Engineering, Washington University, St. Louis, Missouri; ${ }^{2}$ Department of Radiology, Washington \\ University School of Medicine, St. Louis, Missouri; ${ }^{3}$ School of Computer Science, University of Birmingham, Edgbaston, \\ Birmingham, United Kingdom; ${ }^{4}$ Biochemistry and Molecular Biophysics, Washington University School of Medicine, St. Louis, \\ Missouri; and ${ }^{5}$ Department of Physics, Washington University, St. Louis, Missouri
}

Spatial and temporal coregistration of nuclear and optical images can enable the fusion of the information from these complementary molecular imaging modalities. A critical challenge is in integrating the optical and nuclear imaging hardware. Flexible fiber-based fluorescence-mediated tomography (FMT) systems provide a viable solution. The various bore sizes of small-animal nuclear imaging systems can potentially accommodate the FMT fiber imaging arrays. In addition, FMT imaging facilitates coregistration of the nuclear and optical contrasts in time. Herein, we combine a fiber-based FMT system with a preclinical SPECT/CT platform. Feasibility of in vivo imaging is demonstrated by tracking a monomolecular multimodal imaging agent (MOMIA) during transport from the forepaw to the axillary lymph node region of a rat. Methods: The fiber-based, videorate FMT imaging system is composed of 12 sources (785- and 830-nm laser diodes) and 13 detectors. To maintain high temporal sampling, the system simultaneously acquires ratio-metric data at each detector. A 3-dimensional finite element model derived from CT projections provides anatomically based light propagation modeling. Injection of a MOMIA intradermally into the forepaw of rats provided spatially and temporally coregistered nuclear and optical contrasts. FMT data were acquired concurrently with SPECT and CT data. The incorporation of SPECT data as a priori information in the reconstruction of FMT data integrated both optical and nuclear contrasts. Results: Accurate depth localization of phantoms with different thicknesses was accomplished with an average center-of-mass error of $4.1 \pm 2.1 \mathrm{~mm}$ between FMT and SPECT measurements. During in vivo tests, fluorescence and radioactivity from the MOMIA were colocalized in spatially coincident regions with an average center-of-mass error of $2.68 \pm 1.0 \mathrm{~mm}$ between FMT and SPECT for axillary lymph node localization. Intravital imaging with surgical exposure of the lymph node validated the localization of the optical contrast. Conclusion: The feasibility of integrating a fiber-based, video-rate FMT system with a commercial preclinical SPECT/CT platform was established. These coregistered FMT and SPECT/CT results with MOMIAs may facilitate the development of the next gener-

Received Mar. 13, 2012; revision accepted Oct. 18, 2012.

For correspondence or reprints contact: Joseph P. Culver, Department of Radiology, Washington University School of Medicine, 4525 Scott Ave., St. Louis, MO 63110.

E-mail: culverj@wustl.edu

Published online Feb. 27, 2013

COPYRIGHT @ 2013 by the Society of Nuclear Medicine and Molecular Imaging, Inc. ation of preclinical and clinical multimodal optical-nuclear platforms for a broad array of imaging applications and help elucidate the underlying biologic processes relevant to cancer diagnosis and therapy monitoring.

Key Words: multimodal imaging; FMT; nuclear; optical

J Nucl Med 2013; 54:639-646

DOI: 10.2967/jnumed.112.105742

$\mathbf{M}$ ultimodal imaging strives to improve on unimodal imaging by combining multiple contrasts. For example, combining anatomic and molecular imaging (PET/CT and SPECT/CT) has provided an anatomic context for the detailed molecular information from the nuclear contrasts (1-3). However, full fusion of the anatomic and molecular information is limited by the relatively weak connection between the different contrasts. Alternatively, multiple molecular contrasts might be joined through specific links (4-6). For example, optical methods have unique activation contrast mechanisms, which are complementary to PET/SPECT information. The information available to both nuclear and optical contrasts can be harnessed directly using a monomolecular multimodal imaging agent (MOMIA) (4-7). Imaging of MOMIAs requires suitable instrumentation and algorithms. To combine optical data with PET/SPECT data, optical contrasts can be imaged in 3 dimensions by diffuse optical tomography (DOT) (8-13). In this work, we demonstrate the feasibility of combining a previously reported fiber-based, video-rate fluorescencemediated tomography (FMT) system (14) with a preclinical NanoSPECT/CT platform (Bioscan, Inc.) for combined optical and nuclear imaging of dynamic events associated with lymphatic transport.

The design and construction of a multimodal imaging platform presents several challenges. The first is integrating hardware such that each modality can function with minimal, or acceptable, interference from the others. Hardware integration requires compatibility of all the parts for both platforms. The second challenge is spatial and temporal 
coregistration of information from the disparate reporting strategies under each modality. For instance, the distinct chemical and physical characteristics of traditional contrast agents might lead to different biodistribution and migration rates. MOMIAs are being developed to address these pharmacokinetic issues. Recent work has demonstrated the use of this approach for SPECT/optical imaging $(5,15,16)$, PET/optical imaging $(6,7,17)$, and MR/optical imaging (1820 ). The last challenge is determining how to merge the anatomic or functional information from multiple technologies into a single imaging output, which is currently a subject of intensive research, though most of the work is focused on incorporating structural information from radiography or $\mathrm{CT}(8,11,16)$ or MR imaging $(18,21)$ with molecular imaging. Merging information that leverages the respective strengths of each modality would provide new insight into biologic processes relevant to cancer diagnosis and therapy monitoring $(4,11)$.

To address the instrument integration challenges, we took advantage of the flexibility of fiber arrays to provide a compact conduit of light to and from the animal so that the FMT imaging array could fit into the bore of the existing NanoSPECT/CT system without modifications. In addition, to facilitate integration of the nuclear and optical contrasts, we used a MOMIA that was based on a radiolabeled nearinfrared dye synthesized in our laboratory (6).

Feasibility studies of simultaneous fluorescence and radioactive imaging were performed in tissue-simulating phantoms and in vivo in rats. Accurate depth localization of the MOMIA targets was established to depths of up to $10 \mathrm{~mm}$. Fluorescence and radioactivity from an injection of MOMIA into the forepaw region was imaged over time to follow the lymphatic dynamics. These studies were used to assess whether the proposed combined multimodal platform had the potential to become a practical tool for a broad array of imaging applications, ranging from the detection of early disease to the monitoring of disease progress and therapies.

\section{MATERIALS AND METHODS}

\section{Fiber-Based FMT Imaging System}

DOT sources and detector consoles (laser diodes with a wavelength of $785 \mathrm{~nm}$ [DL7140-201S; Thorlabs; $3.5 \mathrm{~mW}$ ] and $830 \mathrm{~nm}$ [HL8325G; Thorlabs; $1.5 \mathrm{~mW}$ ]) have dedicated drivers and control lines for each source to allow flexible software-configurable source encoding (frequency and time encoding). The detection channels use optically filtered discrete avalanche photodiodes (C5460-01; Hamamatsu) digitized with dedicated 24-bit analog-to-digital converters (HD192; Motu). The narrowband optical filters (CVI) have an $830 \pm 10 \mathrm{~nm}$ center wavelength and an out-of-band rejection of OD4, thus blocking the excitation light while passing the fluorescence and reference signals. An aspheric lens was used to collimate the light to optimize the blocking of excitation light by the narrowband interference filter and to enhance the detection of fluorescence signal. With this scheme, we acquired frequency-encoded fluorescence emission and reference transmission (used to normalize the measured fluorescence) light levels concurrently at each detector. These ratio-metric data allow DOT reconstructions using the normalized Born approximation, resulting in a map of quantified fluorochrome distribution. All data were acquired at a frame rate of $30 \mathrm{~Hz}$. One hundred eight measurements from source-detector pairs representing the first, second, and third nearest neighbors were used for image reconstruction. Further design elements and capabilities of the fiber-based, video-rate FMT are detailed in a previous publication (14).

The DOT imaging fiber array is composed of a grid of alternating sources $(n=12)$ and detectors $(n=13)$. Light from the sources is coupled into a 0.5 numeric aperture with 2.5 -mm-diameter fiber bundles made of borosilicate glass sheathed with lightweight silicone. The DOT imaging array, composed of flexible silicone with right-angle fibers, was integrated with the NanoSPECT/CT system.

\section{SPECT/CT Acquisition}

To test the compatibility of inserting our fiber-based FMT into a nuclear platform, we used a NanoSPECT/CT platform. For NanoSPECT/CT, CT was performed first (using a 45-kVp energy tube at $177 \mathrm{~mA}$ and 180 projections, with a 400-ms exposure with a pitch of 1) and then helical SPECT followed (16 projections, with $60 \mathrm{~s}$ each). CT and SPECT projections were reconstructed using InvivoScope software (Bioscan, Inc.). The high-resolution NanoSPECT/CT anatomic and radioactive datasets have an isotropic voxel size of 0.4 and $0.6 \mathrm{~mm}$, respectively.

\section{MOMIA Synthesis}

DOTA-Gly-Ser-Gly-Lys(Cypate)-E-Ahx- $\mathrm{NH}_{2}$, LS444, was synthesized and radiolabeled with ${ }^{111} \mathrm{InCl}_{3}(111 \mathrm{kBq}$ [3.0 $\mu \mathrm{Ci}$; MDSNordion) in aqueous buffer $(190 \mu \mathrm{L}, 0.4 \mathrm{M}$ sodium acetate, $\mathrm{pH}$ $4.5,30 \mathrm{~min}, 98^{\circ} \mathrm{C}$ ) as described previously $(6,7)$. Labeling efficiency and radiochemical purity were checked by high-performance liquid chromatography (Supelcosil ABZ + Plus, HPLC column [Gilson], 15 $\mathrm{cm} \times 4 \mathrm{~mm}, 5 \mu \mathrm{m}$ ), with a gradient of $\mathrm{H}_{2} \mathrm{O}$ and acetonitrile containing $0.1 \%$ trifluoroacetic acid. The radiochemical purities of peptides used in the studies were always above 95\%. The specific activity was $8,251 \mathrm{kBq} / \mathrm{nmol}(223.0 \mu \mathrm{Ci} / \mathrm{nmol})$.

\section{Experimental Protocol}

Animal handling and preparation were performed according to the guidelines approved by the Washington University School of Medicine Animal Studies Committee for humane care and use of laboratory animals. A flowchart of the acquisition timeline is presented in Supplemental Figure 1A (supplemental materials are available online only at http://jnm.snmjournals.org). The rats ( $n=5$; weight, 200-250 g; female Sprague-Dawley [HSD]) were first anesthetized via a mixture of ketamine and xylazine ( 85 and $15 \mathrm{mg} / \mathrm{kg}$ intraperitoneally, respectively). The MOMIA (100 $\mu \mathrm{L}$ of ${ }^{111} \mathrm{In}$-LS444) was then administered via forepaw injection. Concurrent FMT and SPECT/CT scans were obtained immediately after injection.

The DOT imaging fiber array was securely positioned on top of the rat, which was then advanced into the NanoSPECT/CT chamber (Supplemental Fig. 1B). The SPECT/CT regions were selected to include the injection site and the DOT imaging pad using a top-view topogram. CT was performed, followed by helical SPECT, with total acquisition times of 5 and $16 \mathrm{~min}$, respectively. To evaluate the influence of the DOT array on the SPECT/CT datasets, we also acquired SPECT/CT data without the DOT fiber array with the same imaging protocol as described earlier (Supplemental Fig. 1C).

For reference and verification of ${ }^{111}$ In-LS444 uptake by the lymph nodes (LNs), reflectance fluorescence images were acquired 
in vivo before and after removal of overlying skin after euthanasia using the near-infrared reflectance fluorescence system (Pearl; LiCor Biosciences). Fluorescence and brightfield microscopy images of the excised LNs were acquired using a BX51 upright epifluorescence microscope (Olympus America) (supplemental data).

To evaluate the performance of the system as a function of depth, we followed the approach used for the stand-alone FMT system (14) but in this instance with MOMIA targets. The tissuemimicking phantoms were constructed by mixing agarose (SigmaAldrich) with intralipid (20\% fat emulsion; Fresenius Kabi), and India ink (Speedball) to obtain the appropriate absorption and scattering properties $\mu_{\mathrm{a}}=0.19 \mathrm{~cm}^{-1}$ and $\mu^{\prime}{ }_{\mathrm{s}}=3.4 \mathrm{~cm}^{-1}$, respectively. The mixture was poured into a mold and allowed to solidify at room temperature. Targets consisting of 3-mm-diameter plastic tubes filled with the MOMIA were prepared and embedded at 4-, 7-, and $10-\mathrm{mm}$ depths in the tissue-simulating phantoms. The imaging protocols described above were implemented to acquire optical and nuclear datasets of the phantoms with targets at different depths.

\section{Fluorescence DOT Reconstruction}

We generated a subject-specific 3-dimensional (3D) finite element model (FEM) (Fig. 1). Using the anatomic data of rats obtained from 3D CT projections (Fig. 1A), we created a tetrahedral mesh using Mimics (Materialise Inc.) with a maximum internodal distance of $1 \mathrm{~mm}$ both on the surface and within the mesh volume. The mesh, composed of 400,399 linear tetrahedral elements connected by 67,931 nodes, was segmented into 2 tissue types-bone and soft tissues-and the tissue types were assigned their corresponding optical properties $\left(\mu_{\mathrm{a}}=0.17 \mathrm{~cm}^{-1}\right.$, $\mu^{\prime}{ }_{\mathrm{s}}=24 \mathrm{~cm}^{-1}$ and $\mu_{\mathrm{a}}=0.19 \mathrm{~cm}^{-1}, \mu_{\mathrm{s}}^{\prime}=34 \mathrm{~cm}^{-1}$, respectively) (Figs. 1C and 1D, respectively). A heterogeneous tissue model was used because previous studies have shown improvements relative to light models that assume homogeneous optical properties (22-25). For the 2 tissue-simulating phantom imaging, we also used the $3 \mathrm{D}$ phantom $\mathrm{CT}$ projections and created a homogeneous mesh and assigned the corresponding optical properties $\left(\mu_{\mathrm{a}}=0.19 \mathrm{~cm}^{-1}, \mu_{\mathrm{s}}^{\prime}=34 \mathrm{~cm}^{-1}\right)$. A second anatomic CT dataset of each rat and tissue-mimicking phantom was obtained with DOT fibers in place and used to obtain the 3D coordinates of the optode (sources and detectors) positions (Fig. 1B). The resulting FEM mesh and coregistered source and detector locations were used for light modeling (Fig. 1E).
The light transport through the tissue was calculated using finite element software (NIRFAST) following our previously reported methods (26). Briefly, an adjoint Jacobian matrix approach was used to construct a sensitivity matrix. The construction of the sensitivity matrix specific to fluorescence imaging follows the methods previously described (14). The sensitivity matrix maps the relationship between the optical parameters (fluorescence distribution) and the measurements (27) using the normalized Born approach (14). We directly inverted the sensitivity matrix via a Moore-Penrose generalized inverse method $(28,29)$. The experimental source detector measurements were converted into images by matrix multiplication with the inverted matrix. The recovered fluorescence yield of the tissue at each FEM node was then presented in 3 dimensions and the mesh nodes transformed to a $3 \mathrm{D}$ space of $1 \mathrm{~mm}$ (isotropic voxel space) for integration with SPECT/CT data (the point spread function of the FMT system is $\sim 1.2 \mathrm{~cm}$ at a $1-\mathrm{cm}$ depth (14)).

The accuracy of the reconstructed FMT image was further improved using the radioactivity measurement obtained from SPECT data as a spatial priori to create a binary mask. The mask was then multiplied with the simulated light propagation matrix (sensitivity matrix) to constrain the FMT image reconstruction.

\section{Quantification of Influence of FMT Fibers on SPECT and $\mathbf{C T}$}

We evaluated the potential for the presence of optical fibers to negatively affect the imaging performance of the SPECT/CT systems. The influence of the optical fibers on the transmitted $x$-ray depends on the linear attenuation coefficient $(\mu)$ of glass fibers at a given energy window and the thickness of the fiber arrays $(l)$ (Supplemental Fig. 1C). The fraction of the transmitted $\mathrm{x}$-ray beam was calculated using the equation $I=I_{0} e^{-\mu \cdot l}$, where $I_{0}$ and $I$ are the incident and the transmitted x-ray beams, respectively, and $l$ is the effective thickness of the glass fiber elements.

In addition, the normalized root mean square error (NRMSE) was used to quantify the distortions induced in the nuclear data due to the presence of the optical fibers:

$$
\text { NRMSE }=\frac{\sqrt{\frac{\left(X-X_{r e f}\right)^{2}}{N}}}{\max \left(X_{r e f}\right)},
$$

where $X$ is the nuclear image with DOT fiber array, and $X_{\text {ref }}$ is the reference image without the optical fibers. $N$ is the total number of

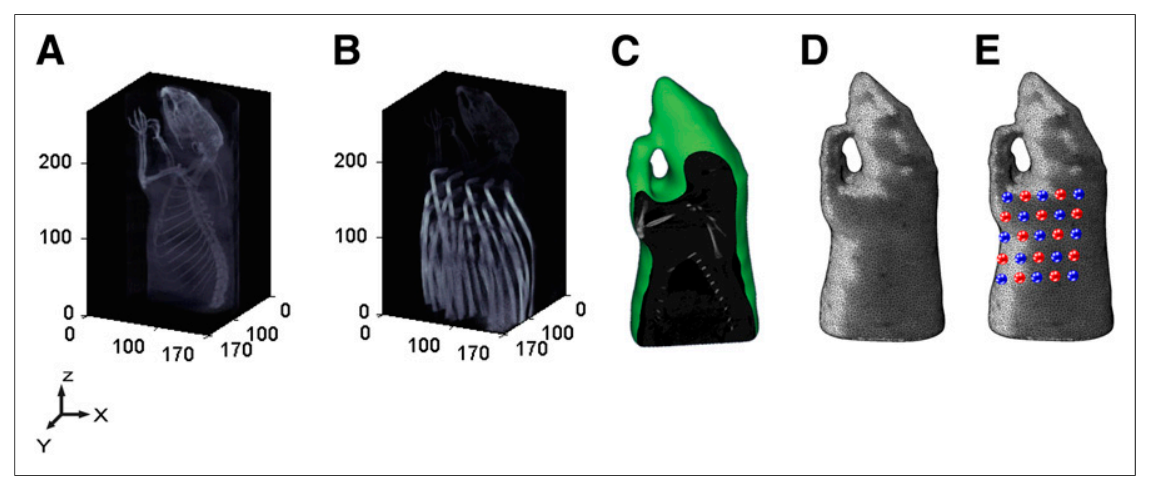

FIGURE 1. Fluorescence DOT image reconstruction. (A) CT scan is used to capture 3D structure of anatomy of rat. (B) CT image with fiber array of DOT used to obtain optode positions. (C) Sagittal section of anatomic CT image after segmentation into bone and soft-tissue region using Mimics. (D) 3D FEM of small-animal half-body mesh generated from CT within Mimics to be used for forward modeling of light propagation. (E) Small-animal mesh after projection of optodes (source [red] and detector [blue]). Sensitivity matrix is then generated using mesh and measurement parameters as main inputs for FEM representation of light in tissue using NIRFAST (Dartmouth). 
image pixels. NRMSE is computed for both the SPECT and the CT datasets.

\section{Quantifying Image Quality: Localization Accuracy}

The localization accuracy of the FMT reconstruction, compared with its corresponding SPECT datasets, was evaluated by calculating the absolute error in the center of masses (COMs) (of the optical and nuclear contrasts) of the $\mathrm{LN}$ volumes. The mean fluorescence and radioactivity intensity values in the $x, y, z$ planes were computed for each subject from the reconstructed nuclear and optical datasets to find the COMs. In addition, the absolute error of the COMs was computed to assess the positional error between the fluorescence and radioactive distributions. The COMs and COM errors of the reconstructed MOMIA tubes were also computed by averaging the fluorescence and radioactive intensity values for each experimental depth. In addition, the difference in spatial resolution was quantified by comparing the volumetric ratio of the regions of interest between the nuclear and optical datasets of the various depths of the reconstructed tubes.

\section{RESULTS}

The goal of this study was to demonstrate the feasibility of combining fiber-based FMT with NanoSPECT/CT. This combined nuclear-optical platform obtained data from both contrast mechanisms concurrently in space and time. Signal from a MOMIA, ${ }^{111}$ In-LS444, was used to facilitate the spatial integration of the nuclear and optical data.

The potential influence of the optical fibers on the nuclear imaging datasets was evaluated both by computing the x-ray beam attenuation due to glass fibers and, more directly, by measuring the variance introduced into the CT and SPECT signals by the DOT fiber array. The experimentally derived transmission losses for the x-ray beam were consistent with the trends of the theoretic estimates based on attenuation coefficients (energy-dependent) of the glass fiber (Table 1). Model calculations estimate the $\mathrm{x}$-ray beam transmission through the fiber array to be $31 \%$ for CT $(45 \mathrm{keV})$ and $68 \%$ for SPECT $(245 \mathrm{keV})$.

The computation of the NRMSE also demonstrated the existence of distortion introduced by the optical fibers (Fig. 2A) in the nuclear data. The NRMSE between the CT anatomic data with and without FMT imaging fibers (Figs. 2B and $2 \mathrm{C}$, respectively) is $8.5 \%$. The NRMSE between the radioactive datasets acquired in the presence (Fig. 2D) and absence (Fig. 2E) of fibers is found to be $3.1 \pm 1.3 \%$, which also accounts for the additional MOMIA accumulated in LNs during the process of acquiring the reference SPECT measurements. For improved visual reference, in the remainder of the article the fluorescence and radioactive data are coregistered and displayed on anatomic CT images obtained after removal of the DOT fiber arrays (Fig. 2E).

Images of the MOMIA targets in tissue-mimicking phantoms confirm the localization of the targets in the range of the depths from 5 to $10 \mathrm{~mm}$ (Supplemental Fig. 3; Supplemental Tables 1 and 2). The average absolute error between the COM of the optical and nuclear datasets for all 3 depths was $4.1 \pm 2.1 \mathrm{~mm}$. The average depth-dependent spread of the volume of the FMT data, compared with the SPECT data, was $2.4 \pm 0.95$ after thresholding at $30 \%$ maximum.

The coregistered molecular information with its corresponding anatomic structure (Fig. 3) demonstrates the feasibility of obtaining measurements with FMT and SPECT molecular contrasts and structural information with CT. An anatomic CT image of a rat provided structural information that facilitated segmentation of different tissue types, including bone and soft tissue. The tissue-segmented structure was used to generate a finite element mesh, and the corresponding optical properties of the different tissue types were assigned to each FEM to provide an accurate light propagation model for the FMT forward calculation (Fig. 1).

Reconstructed FMT images show uptake of the MOMIA by the LNs in the axillary region (Fig. 3A). The localized radioactive signal in the axillary region of the SPECT/CT image also demonstrated the uptake of the MOMIA by the axillary LNs (Fig. 3B). The highly radioactive signals on the left forepaw, at the injection site, show the starting point of the lymph tract that leads to the axillary nodes. Because of the limited field of view of the FMT fiber array, the injection site is not visible in the reconstructed FMT image.

The uptake of ${ }^{111}$ In-LS444 by the axillary LNs was further validated by combining the SPECT and FMT datasets. The coregistered FMT and SPECT/CT images (Fig. 4A) demonstrate colocalization of the multimodal agent in a spatially coincident region. The colocalization confirms that the radioactive and fluorescence signals originate from the same location corresponding to the LNs. The low overlap percentage can be attributed to the difference in resolution (or point-spread function) between the optical and nuclear imaging systems and the presence of fractional component of nonradiolabeled LS444. However, the free LS444 fractional component is expected to be minimal because of the high radiochemical purities of peptides used.

TABLE 1

Evaluation Effect of Fluorescence DOT Fiber Arrays on Transmitted X-Ray Beams of Nuclear Imaging System

\begin{tabular}{|c|c|c|}
\hline Parameter & SPECT & CT \\
\hline Radiation energy (E) & $245 \mathrm{keV}\left({ }^{111} / \mathrm{n}\right)$ & $45 \mathrm{keV}$ \\
\hline Attenuation coefficient of glass, $\mu(E), \mathrm{cm}^{-1}$ & 0.256 & 0.781 \\
\hline Thickness $(I)$ in $\mathrm{cm}$ & 1.5 & 1.5 \\
\hline Transmission (\%) & 68 & 31 \\
\hline
\end{tabular}




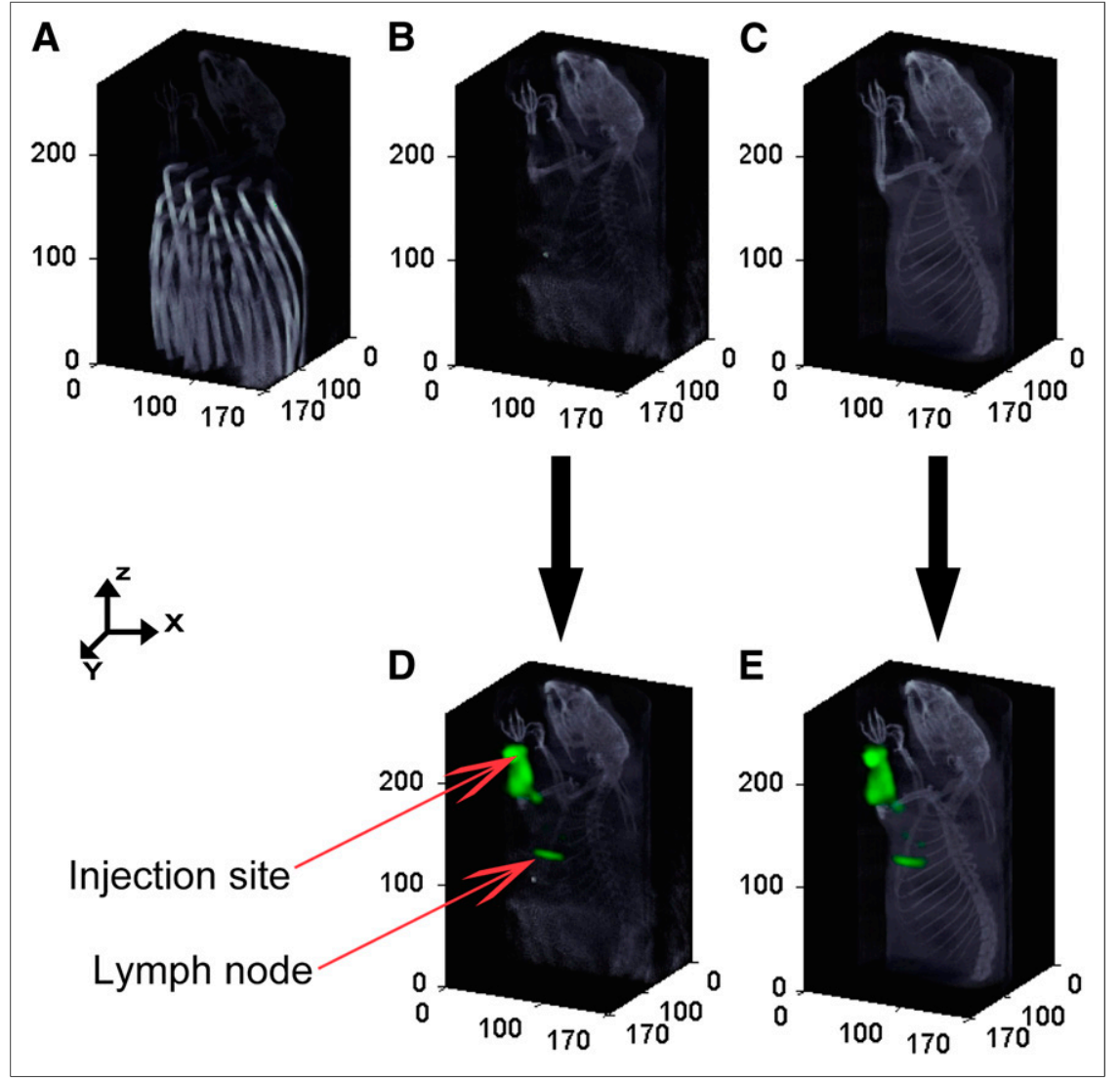

FIGURE 2. Evaluating influence of fiber arrays on SPECT and CT images: CT image of rat with fiber array of DOT (A), CT image of same rat shown after voxels related to fiberarray of DOT were removed (B), CT image acquired after DOT imaging pad was removed (C). Anatomic structures acquired without fibers are used to display fluorescence and radioactive distribution for all 5 rats. (D) SPECT data were acquired in presence of DOT fiber array in rat after injection of MOMIA ${ }^{111}$ In-LS444 into left forearm. (E) Shown are SPECT data of same rat acquired after fibers were removed.
Having demonstrated the colocalization of the fluorescence and radioactive distribution, we explored a simple method to incorporate the high-resolution NanoSPECT/ $\mathrm{CT}$ radioactive data into the DOT image reconstruction to improve the localization accuracy of the axillary LN signal. A binary mask, created from the radioactivity distribution of the SPECT data, was multiplied by the simulated light propagation matrix to constrain the FMT image reconstruction. The SPECT/CT measurements fused with the FMT datasets reconstructed with a priori information (Fig. 4B) demonstrated an improvement in congruency of the radioactive and fluorescence signal in the axillary regions. Because SPECT was used as a hard prior, the overlap between DOT and SPECT is inherently unity. Likewise, it is assumed that the fluorescence signal originates from conjugated complexes and not from free optically active fragments. A soft-prior approach to relax these conditions is suggested below. The average spatial discrepancy between the COM of the optical-nuclear datasets for all 5 rats was $2.68 \pm 1.0$ and $1.33 \pm 0.85$ $\mathrm{mm}$, respectively, before and after the incorporation of SPECT as a priori information.

\section{DISCUSSION}

The multimodal optical-nuclear platform shown has the potential to elucidate underlying biologic mechanisms relevant to a wide array of diseases. We established the feasibility of integrating a fiber-based, video-rate FMT system with a preclinical NanoSPECT/CT platform. We used our recently developed MOMIA, which has the unique structural feature that both signals (fluorescence and radioactivity) emanate from the same source. The near-infrared fluorescent molecular probe LS444 served as a contrast agent for FMT, and the ${ }^{111}$ In served as a source of signal for SPECT to facilitate the fusion of the optical and nuclear datasets with high spatial precision.

In this study, we evaluated the FMT and SPECT/CT system for imaging LNs in rats. CT provided highresolution, coregistered anatomic images. The combined optical-nuclear imaging demonstrated colocalization of a MOMIA in a spatially coincident region. We further demonstrated the congruency of the colocalization by incorporating SPECT as a priori information in the DOT reconstruction. The average spatial discrepancy in the COMs of the contrasts between SPECT and FMT improved from $2.68 \pm 1.0$ to $1.33 \pm 0.85 \mathrm{~mm}$ after SPECT was incorporated into the into the FMT reconstruction.

Datasets acquired separately with optical and nuclear platforms have been integrated previously $(12,13)$. For instance, Nahrendurfet et al. demonstrated the similarity between FMT and PET by combining the 2 datasets acquired sequentially on 2 different scanners using fiducial markers to coregister the data (13). A strength of the current simultaneous optical-nuclear system is that it avoids the potential misalignment of datasets due to involuntary nonuniform movement of tissues during repositioning. Furthermore, the 


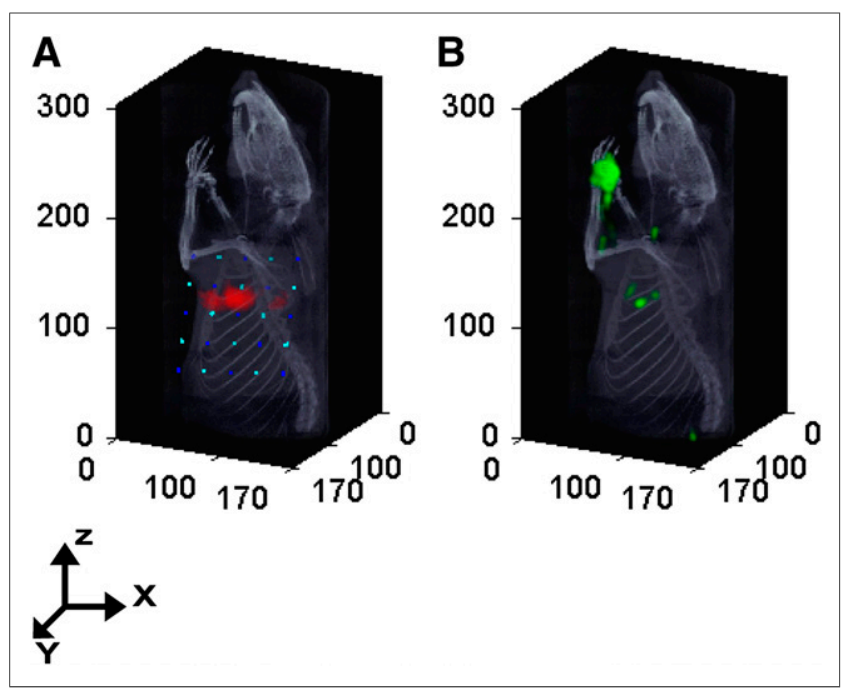

FIGURE 3. Representative sentinel LN mapping using optical and nuclear imaging systems. (A) DOT/CT image of fluorescent LNs shown at 2-mm depth in rat after injection of multimodal imaging agent ${ }^{111}$ In-LS444 into left forearm. (B) SPECT/CT image demonstrating localization of axillary $L N$ identified by accumulation of MOMIA.

datasets in the presented study are coregistered in time. The first small-animal study in which optical imaging (scan rate not reported) was physically integrated into a PET system was reported by $\mathrm{Li}$ et al. (12). Localization of the tumor with simultaneously acquired nuclear and optical datasets was performed after injection of 2 separate contrast agents (for fluorescence and radioactive detections) at different time points, separated by $24 \mathrm{~h}$; thus, the datasets were not coregistered in time. Further, acquisition of the anatomic information using a different scanner might lead to a coregistration error of the functional molecular data with reference anatomy. To a significant extent, the system presented in this paper addresses many of these difficulties by combining 3 modalities (FMT, SPECT, and CT) within a single device. The FMT and SPECT/CT platform acquires the different functional (fluorescence and radioactive) and anatomic data either simultaneously or sequentially without requiring the subject to be moved from the bed. This design essentially eliminates differences in subject positioning and minimizes misalignment due to involuntary internal organ motion.

Although the current study did not focus on the dynamics of the LN accumulation, we have previously reported the capability of the FMT system for dynamic imaging by monitoring and generating time course data of the lymphatic dynamics for indocyanine green (14). Accumulated dye in the axillary region of rats was imaged to a depth of 10-12 $\mathrm{mm}$ over a 10-min time course. Future studies will be needed to explore dynamic imaging of pharmacokinetics and pharmacodynamics for multimodal contrast agents.

Optical imaging can leverage the SPECT and CT information to improve accuracy during data processing and image reconstruction $(10,30)$. For instance, we generated a small-animal 3D FEM using the 3D anatomic projections obtained from a CT scan to improve forward modeling of light propagation. In addition, incorporating SPECT data into the FMT reconstruction augmented the localization accuracy. An important future area of work is in developing algorithms to incorporate the SPECT data as a soft prior in the FMT reconstruction to optimize the fusion of the 2 data types (10).

The current FMT system setup has high dynamic range and linear response and accurate localization at various depths as shown with phantom studies. However, several potential improvements of the fiber-based, video-rate FMT system can still be identified. For instance, the sensitivity of the optical imaging in general is expected to decline with imaging depth, whereas SPECT has no depth limitation and easily extends to whole-body imaging. Possible extensions on the current design include extending the FMT field of view for more complete coverage. The design of the DOT imaging array shape and size can be based on the intended application. For example, the imaging array could have a cylindric shape with extended sources and detectors for whole-body imaging. The potential for an expanded fiber array system with up to 48 sources and 48 detectors has been demonstrated for brain imaging in humans (31). A higher density imaging array could also potentially increase the resolution, particularly at the shallower depths for accurate localization of the target (32). A second limitation is the existence of distortion or artifacts on the CT images due to the high attenuation coefficient of borosilicate glass fibers, compared with the bone. The possibility that switching to optical fibers composed of materials with lower attenuation than bone would reduce the artifacts remains a question for future investigation.

Another area of future work is to leverage the complementary contrast mechanisms of SPECT and FMT to evaluate biologic mechanisms. MOMIAs that use activatable optical contrast mechanisms provide complementary information to the always-on SPECT information. SPECT can be used for whole-body imaging to localize diseased tissue on the basis of maps of the concentration of molecule-targeted imaging agents whereas FMT would report local molecular events, such as enzymatic activity, to monitor therapeutic response $(5,15)$.

\section{CONCLUSION}

We demonstrated integration of a fiber-based, video-rate FMT system with a preclinical NanoSPECT/CT platform. Because of the flexibility of the imaging fiber array, the video-rate FMT can accommodate the various imaging bore sizes of nuclear imaging systems. We used a MOMIA such that the nuclear and optical signals emanated from the same regions to facilitate the fusion of both datasets with high spatial precision. The depth-profiling capability of FMT was confirmed in phantoms by localizing MOMIA targets in tissue-mimicking phantoms in a range of depths from 5 to $10 \mathrm{~mm}$. The average absolute error between the COM of the optical and nuclear datasets for all 3 depths was $4.1 \pm$ $2.1 \mathrm{~mm}$, and the depth-dependent spread of the volume of the FMT data, compared with SPECT data, was $2.4 \pm 0.95$ 


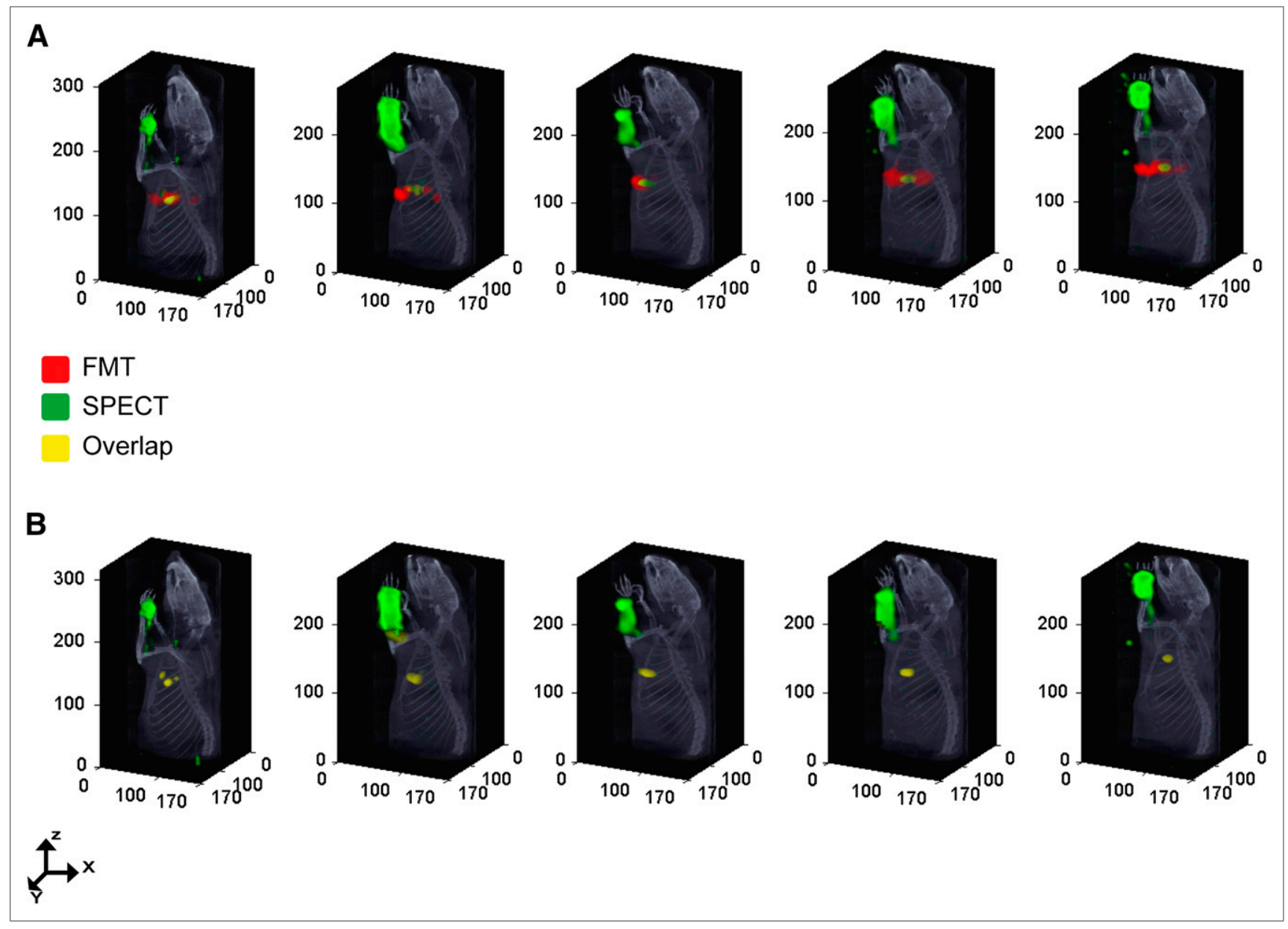

FIGURE 4. Multimodal sentinel LN imaging. (A) Coregistered FMT and SPECT/CT image demonstrates colocalization of MOMIA in spatially coincident region in 5 rats. (B) Robust colocalization on coregistered FMT and SPECT/CT image is demonstrated after SPECT as a priori information was used.

after thresholding at 30\% maximum. For in vivo imaging, we used the anatomic CT datasets to generate 3D FEM for light propagation. Colocalization of the MOMIA in both the FMT and the SPECT contrasts was demonstrated. We also observed improvement in the spatial correlation of the coregistered datasets after incorporating SPECT as a priori information to constrain the FMT data reconstruction. The average spatial discrepancy of the LN COMs between FMT and SPECT improved from $2.68 \pm 1.0$ to $1.33 \pm 0.85 \mathrm{~mm}$ after the SPECT data were incorporated as a priori information into the FMT reconstructions. These results suggest that integrated multimodal FMT and SPECT/CT has the potential to become a powerful and practical tool for a broad array of real-time imaging applications.

\section{DISCLOSURE}

The costs of publication of this article were defrayed in part by the payment of page charges. Therefore, and solely to indicate this fact, this article is hereby marked "advertisement" in accordance with 18 USC section 1734. This research was supported in part by the Network for Translational Research U54CA136398 TSP-3, R01EB009233, R01EB008111, and U54CA136398 TSP-1 and R01EB008458. In addition, the study was supported by grant K01RR026095. No other potential conflict of interest relevant to this article was reported.

\section{REFERENCES}

1. Alberini JL, Edeline V, Giraudet AL, et al. Single photon emission tomography/ computed tomography (SPET/CT) and positron emission tomography/computed tomography (PET/CT) to image cancer. J Surg Oncol. 2011;103:602-606.

2. Even-Sapir E, Keidar Z, Bar-Shalom R. Hybrid imaging (SPECT/CT and PET/ CT)-improving the diagnostic accuracy of functional/metabolic and anatomic imaging. Semin Nucl Med. 2009;39:264-275.

3. Patton JA, Townsend DW, Hutton BF. Hybrid imaging technology: from dreams and vision to clinical devices. Semin Nucl Med. 2009;39:247-263.

4. Achilefu S, Ye YP, Bloch S, Zhang ZR, Berezin M, Liang KX. Development of contrast effectors for optical and multimodal imaging of tumors. Abstr Pap Am Chem S. 2004;228:U824-U824.

5. Edwards WB, Akers WJ, Ye Y, et al. Multimodal imaging of integrin receptorpositive tumors by bioluminescence, fluorescence, gamma scintigraphy, and singlephoton emission computed tomography using a cyclic RGD peptide labeled with a near-infrared fluorescent dye and a radionuclide. Mol Imaging. 2009;8:101-110.

6. Zhang Z, Liang KX, Bloch S, Berezin M, Achilefu S. Monomolecular multimodal fluorescence-radioisotope imaging agents. Bioconjug Chem. 2005;16:1232-1239.

7. Zhang Z, Achilefu S. Spectral properties of pro-multimodal imaging agents derived from a NIR dye and a metal chelator. Photochem Photobiol. 2005;81:14991504. 
8. Barber WC, Lin Y, Nalcioglu O, Iwanczyk JS, Hartsough NE, Gulsen G. Combined fluorescence and $\mathrm{x}$-ray tomography for quantitative in vivo detection of fluorophore. Technol Cancer Res Treat. 2010;9:45-52.

9. Cao L, Breithaupt M, Peter J. Geometrical co-calibration of a tomographic optical system with CT for intrinsically co-registered imaging. Phys Med Biol. 2010;55:1591-1606.

10. Cao L, Peter J. Bayesian reconstruction strategy of fluorescence-mediated tomography using an integrated SPECT-CT-OT system. Phys Med Biol. 2010;55:2693-2708.

11. Culver J, Akers W, Achilefu S. Multimodality molecular imaging with combined optical and SPECT/PET modalities. J Nucl Med. 2008;49:169-172.

12. Li C, Yang YF, Mitchell GS, Cherry SR. Simultaneous PET and multispectral 3dimensional fluorescence optical tomography imaging system. J Nucl Med. 2011;52:1268-1275.

13. Nahrendorf M, Keliher E, Marinelli B, et al. Hybrid PET-optical imaging using targeted probes. Proc Natl Acad Sci USA. 2010;107:7910-7915.

14. Solomon M, White BR, Nothdruft RE, et al. Video-rate fluorescence diffuse optical tomography for in vivo sentinel lymph node imaging. Biomed Opt Express. 2011;2:3267-3277.

15. Lee H, Akers WJ, Cheney PP, et al. Complementary optical and nuclear imaging of caspase- 3 activity using combined activatable and radio-labeled multimodality molecular probe. J Biomed Opt. 2009;14:040507.

16. Zhang Q, Brukilacchio TJ, Li A, et al. Coregistered tomographic x-ray and optical breast imaging: initial results. J Biomed Opt. 2005;10:024033.

17. Berezin MY, Guo K, Teng B, et al. Radioactivity-synchronized fluorescence enhancement using a radionuclide fluorescence-quenched dye. J Am Chem Soc. 2009;131:9198-9200.

18. Gulsen G, Yu H, Wang J, et al. Congruent MRI and near-infrared spectroscopy for functional and structural imaging of tumors. Technol Cancer Res Treat. 2002;1:497-505.

19. Guo K, Berezin MY, Zheng J, et al. Near infrared-fluorescent and magnetic resonance imaging molecular probe with high T-1 relaxivity for in vivo multimodal imaging. Chem Commun (Camb). 2010;46:3705-3707.

20. Ntziachristos V, Yodh AG, Schnall MD, Chance B. MRI-guided diffuse optical spectroscopy of malignant and benign breast lesions. Neoplasia. 2002;4:347-354.
21. Barbour RL, Graber HL, Chang JW, Barbour SLS, Koo PC, Aronson R. MRIguided optical tomography: prospects and computation for a new imaging method. Ieee Comput Sci Eng. Win. 1995;2:63-77.

22. Niedre MJ, Turner GM, Ntziachristos V. Time-resolved imaging of optical coefficients through murine chest cavities. J Biomed Opt. 2006;11:064017.

23. Dehaes M, Grant PE, Sliva DD, et al. Assessment of the frequency-domain multi-distance method to evaluate the brain optical properties: Monte Carlo simulations from neonate to adult. Biomed Opt Express. 2011;2:552-567.

24. Heiskala J, Pollari M, Metsaranta M, Grant PE, Nissila I. Probabilistic atlas can improve reconstruction from optical imaging of the neonatal brain. Opt Express. 2009; 17:14977-14992.

25. Eggebrecht AT, White BR, Ferradal SL, et al. A quantitative spatial comparison of high-density diffuse optical tomography and fMRI cortical mapping. Neuroimage. 2012;61:1120-1128.

26. Dehghani H, Srinivasan S, Pogue BW, Gibson A. Numerical modelling and image reconstruction in diffuse optical tomography. Philos Transact A Math Phys Eng Sci. 2009;367:3073-3093.

27. Ntziachristos V, Weissleder R. Experimental three-dimensional fluorescence reconstruction of diffuse media by use of a normalized Born approximation. Opt Lett. 2001;26:893-895.

28. Culver JP, Siegel AM, Stott JJ, Boas DA. Volumetric diffuse optical tomography of brain activity. Opt Lett. 2003;28:2061-2063.

29. Culver JP, Durduran T, Furuya D, Cheung C, Greenberg JH, Yodh AG. Diffuse optical tomography of cerebral blood flow, oxygenation, and metabolism in rat during focal ischemia. J Cereb Blood Flow Metab. 2003;23:911-924.

30. Li A, Miller EL, Kilmer ME, et al. Tomographic optical breast imaging guided by three-dimensional mammography. Appl Opt. 2003;42:5181-5190.

31. White BR, Snyder AZ, Cohen AL, et al. Resting-state functional connectivity in the human brain revealed with diffuse optical tomography. Neuroimage. 2009;47:148-156.

32. Culver JP, Ntziachristos V, Holboke MJ, Yodh AG. Optimization of optode arrangements for diffuse optical tomography: a singular-value analysis. Opt Lett. 2001;26:701-703. 